\title{
Avaliação do comportamento hidrodinâmico de reator anaeróbio de manta de lodo e fluxo ascendente com diferentes configurações do sistema de distribuição do afluente utilizando fluidodinâmica computacional
}

\author{
Hydrodynamic performance evaluation of an upflow anaerobic \\ sludge blanket reactor with different configurations of the influent \\ distribution system using computational fluid dynamics
}

Diego Bongiorno Cruz', Eudes José Arantes', Karina Querne de Carvalho², Fernando Hermes Passig ${ }^{2}$, Cristiane Kreutz', Morgana Suszek Gonçalves'

\begin{abstract}
RESUMO
Compreender o comportamento hidrodinâmico de reatores biológicos pode auxiliar na detecção de problemas associados a falhas operacionais e de projeto, situações que prejudicam a eficiência do tratamento. Neste artigo, realizaram-se simulações da fluidodinâmica computacional (CFD) de escoamento de duas fases sólida-líquida de um reator anaeróbio de manta de lodo e fluxo ascendente (UASB), em escala piloto (160 L), com tempo de detenção hidráulica (TDH) de 10 h e vazão de 16 L.h?. Um modelo Euler-Euler simplificado foi formulado para simular o comportamento hidrodinâmico da zona de reação, influenciada pela configuração do sistema de distribuição do afluente. Foram avaliadas quatro configurações do sistema de distribuição do afluente no reator: uma entrada na parte central (1) e duas entradas centrais (2), de fluxo ascendente; duas entradas nas laterais (3), de fluxo radial; e três entradas de fluxo descendente (4), utilizando geometrias bidimensionais e tridimensionais para verificar a formação de zonas mortas, curtos-circuitos hidráulicos e caminhos preferenciais. As melhores características hidrodinâmicas e a melhor distribuição do afluente foram verificadas na configuração 4, com melhor perfil de mistura do lodo com a fase líquida, na comparação com as demais configurações. Foi notada formação de vórtices na parte inferior do reator com maior concentração do lodo anaeróbio nessa configuração e de caminhos preferenciais nas laterais do reator na configuração 3, indicando mistura ineficiente do afluente com o lodo anaeróbio. O modelo demonstrou que a configuração do sistema de distribuição do afluente influencia significativamente o comportamento hidrodinâmico do reator UASB.

Palavras-chave: hidrodinâmica; tempo de detenção hidráulica; fluidodinâmica computacional; modelo Euler-Euler; anomalias.
\end{abstract}

\begin{abstract}
Understanding the hydrodynamics behavior of biological reactors can help in the detection of problems related to operational failures and design that adversely affect the efficiency of the treatment. In this paper, computational fluid dynamics (CFD) simulations of two-phase liquid-solid flow were carried out in an upflow anaerobic sludge blanket reactor in pilot scale $(160 \mathrm{~L})$, with hydraulic retention time (HRT) of 10 $\mathrm{h}$ and flowrate 16 L.h ${ }^{-1}$. The Euler-Euler approach was formulated to simulate the reaction zone hydrodynamics. Four configurations of the influent distribution system in the reactor were evaluated: one central inlet (1) and two central inlets (2), upflow; two lateral inlets (3), radial flow; and three inlets, downflow (4), using two and three-dimensional geometries to verify the formation of dead zones, hydraulic short circuiting and preferential pathways. Better influent distribution and greater mixture profile of the sludge with the liquid phase were found in the configuration 4 , compared to the others by the formation of vortices in the bottom part of the reactor with higher concentration of anaerobic sludge. Formation of preferential pathways was noted in the lateral inlets of the reactor in the configuration 3, indicating an inefficient mixture of the influent with the sludge. The model demonstrates that the configuration of the influent distribution system significantly influences the hydrodynamics behavior of the UASB reactor
\end{abstract}

Keywords: hydrodynamics; hydraulic retention time; computational fluid dynamics; Euler-Euler approach; anomalies.

Universidade Tecnológica Federal do Paraná (UTFPR) - Campo Mourão (PR), Brasil. 2UTFPR - Curitiba (PR), Brasil.

Endereço para correspondência: Eudes José Arantes - Universidade Tecnológica Federal do Paraná (UTFPR) - Via Rosalina Maria dos Santos, 1233, CEP: 87301-899 - Campo Mourão (PR), Brasil - E-mail: eudesarantes@utfpr.edu.br

Recebido: 28/11/13 - Aceito: 15/03/16 - Reg. ABES: 127299. 


\section{INTRODUÇÃO}

O comportamento hidrodinâmico de reatores biológicos empregados no tratamento de águas residuárias pode influenciar na velocidade das reações, por meio de alterações na taxa de transferência de massa e na distribuição das reações ao longo dos reatores. Como consequência da distribuição da biomassa e do encadeamento das reações bioquímicas, há diferentes regiões do reator, com composições distintas, em função do tipo de escoamento imposto.

Com estudos detalhados sobre a hidrodinâmica de reatores, como os reatores anaeróbios de manta de lodo e fluxo ascendente (tipo upflow anaerobic sludge blanket - UASB), é possível detectar problemas associados a falhas operacionais e de projeto, desenvolver modelações matemáticas para caracterização do escoamento e comparar variadas configurações dessas unidades.

A caracterização do regime de escoamento em reatores é definida pela distribuição do tempo de residência (DTR). A importância da determinação das curvas de DTR reside no fato de que é possível estabelecer os regimes do fluxo de escoamento, determinar os parâmetros hidrodinâmicos e identificar anomalias no escoamento, tais como zonas mortas, caminhos preferenciais e curtos-circuitos hidráulicos. Essas anomalias podem prejudicar a eficiência dos reatores aplicados no tratamento de águas residuárias, por conta da diminuição do volume útil e do tempo de detenção hidráulica necessário ao desempenho da atividade microbiana (CARVALHO et al., 2008; LEVENSPIEL, 2000).

Além disso, Fogler (1999) afirma que o conhecimento das curvas de DTR permite obter modelagem cinética mais precisa e realizar comparação mais aprofundada do regime hidráulico nas diferentes zonas do reator, sendo assim uma ferramenta útil à otimização dos processos de tratamento.

Modelos matemáticos com descrições de variáveis hidrodinâmicas em geometrias bidimensionais e tridimensionais de reatores têm sido comumente reportados na literatura técnico-científica (MIRANDA et al., 2010). Entre eles, destaca-se a fluidodinâmica computacional, ou computational fluid dynamics (CFD), que é uma ferramenta matemática aplicada à dinâmica dos fluidos para qualquer tipo de escoamento, a fim de facilitar a compreensão de um sistema real, por intermédio da construção de um modelo computacional representativo baseado em equações matemáticas que representam o escoamento de fluidos, com condições de contorno predeterminadas (VERSTEEG \& MALALASEKERA, 1995).

A ferramenta CFD envolve a resolução numérica das equações de conservação de massa, momento e energia do escoamento dos fluidos em uma determinada geometria, com equações adicionais que refletem o problema estudado, em menor escala de tempo e com maior robustez e precisão. Segundo Simões et al. (2009) e Islabão et al. (2010), essa ferramenta pode ser utilizada para simular o comportamento de escoamentos, a transferência de calor, a distribuição da velocidade, a pressão, a temperatura e outros processos físicos em dado sistema.

Historicamente, a CFD vem sendo utilizada há mais de 20 anos como uma ferramenta computacional empregada na modelagem do transporte de fluidos em sistemas de tratamento de águas residuárias. Com a sua evolução, a comunidade científica tem aceitado sua aplicação para análise de processos envolvendo uma unidade operacional que contempla múltiplas fases e características físicas, químicas e biológicas, como é o caso dos reatores tipo UASB (LAURENT et al., 2014; LÓPEZ-JIMÉNEZ et al., 2015).

Por outro lado, poucos estudos são reportados na literatura sobre a aplicação das ferramentas de CFD para simulação de reatores, com destaque para Ren et al. (2009) e Wang et al. (2009). Ren et al. (2009) verificaram similaridade no padrão de escoamento de reatores UASB em diferentes escalas e diminuição do coeficiente de dispersão do escoamento ao longo da altura, da base para o topo. Já Wang et al. (2009) observaram a influência da fase gasosa (movimento, rompimento e alteração do tamanho das bolhas de biogás) na fase sólida, por meio da distribuição da velocidade e do movimento das partículas do lodo em um reator de leito expandido, na produção de bio-hidrogênio. Além disso, esses autores também notaram a mesma influência na fase líquida, com recirculação interna, em razão do aumento da velocidade do escoamento próxima à parede do reator.

Por sua vez, Wang et al. (2010) salientaram que o controle do tempo de detenção hidráulica (TDH) foi o fator-chave na produção de bio-hidrogênio de um reator de leito expandido, pela relação qualitativa com a hidrodinâmica do reator demonstrada pelo modelo CFD.

Considerando que o emprego da CFD pode ser útil para avaliar a eficiência da mistura de fases no reator UASB, constatar possíveis desvios de idealidade no escoamento e possibilitar a otimização do processo, o objetivo deste trabalho foi avaliar as características hidrodinâmicas e a formação de zonas mortas e de caminhos preferenciais para quatro diferentes configurações de sistemas de distribuição do afluente em um reator UASB, utilizando, para isso, a CFD.

\section{METODOLOGIA}

\section{Reator anaeróbio de manta de lodo e fluxo ascendente em escala piloto}

As simulações foram realizadas com base na geometria de um reator UASB, em escala piloto, confeccionado em policloreto de vinila (PVC), com 1,70 m de altura, 0,28 $\mathrm{m}$ de diâmetro e volume total de 160 L. O reator foi operado com tempo de detenção hidráulica (TDH) de 10 h e vazão afluente de 16 L.h ${ }^{-1}$. Informações detalhadas sobre o início da operação e o desempenho desse reator tratando esgotos sanitários, no estado de equilíbrio dinâmico aparente, são descritas por Carvalho et al. (2008). 


\section{Geometrias propostas para a simulação}

Neste trabalho foi adotada abordagem simplificada com divisão do volume do reator em zonas correspondentes ao estado funcional, considerando apenas o sistema de distribuição afluente, o leito de lodo e a zona de digestão, para realização de teste prévio de sensibilidade (Figura 1).

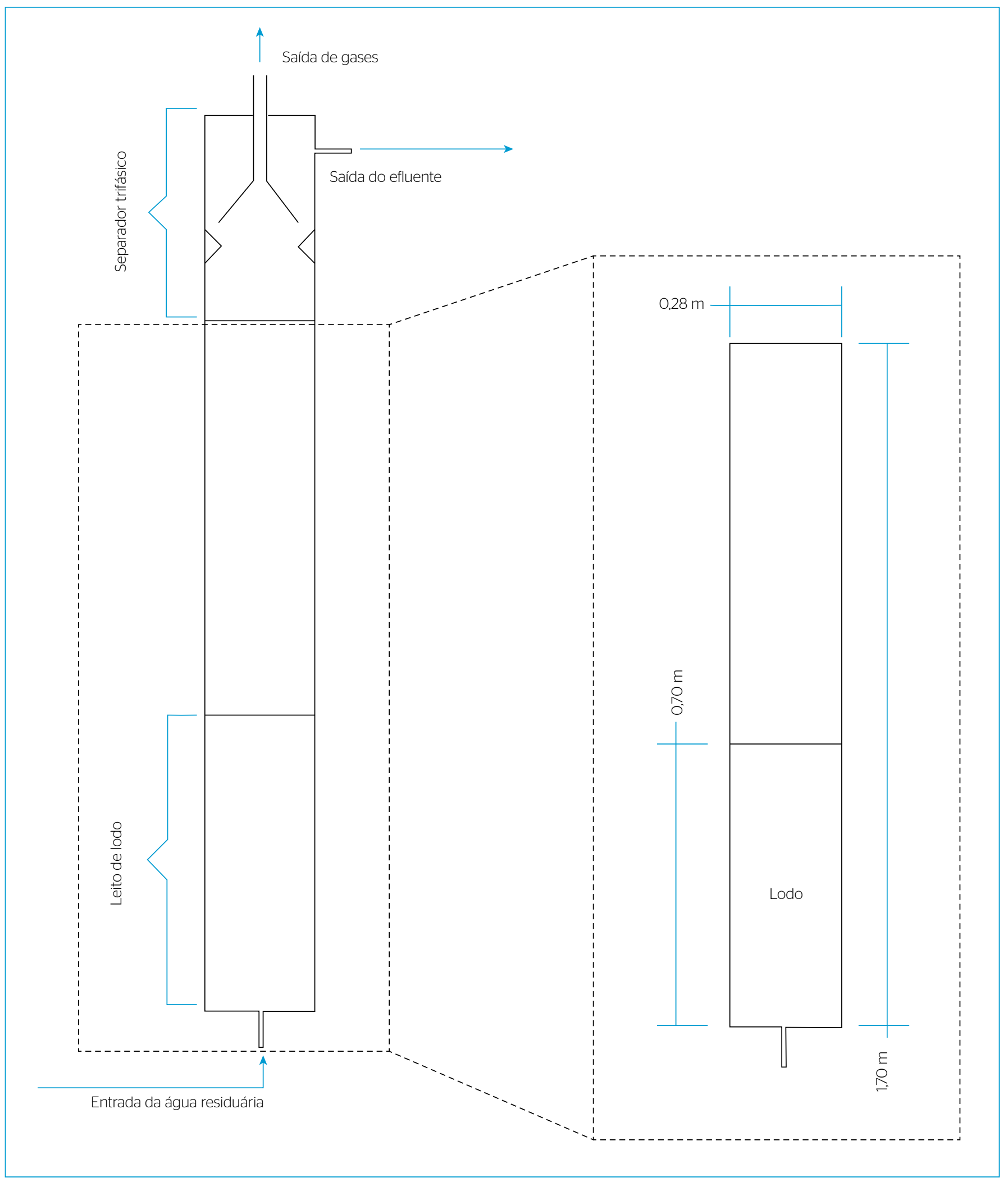

Figura 1 - Desenho esquemático do reator anaeróbio de manta de lodo e fluxo ascendente e desenho do modelo computacional simplificado (detalhe). 
Não foi levada em conta a zona correspondente ao separador trifásico, pois, de acordo com Maestri \& Gimenez (1998), qualquer abordagem que incluísse todos os fatores intervenientes no escoamento de um reator UASB acarretaria grau elevado de complexidade na análise dimensional, tornando-a inútil.

A abordagem desta pesquisa não contempla estudo experimental para calibração e validação dos dados, mas permite uma análise de cenários práticos para a investigação científica prévia das características hidrodinâmicas.

A geometria do reator utilizado como base para este trabalho foi simplificada para demonstrar apenas o sistema de distribuição do afluente e o leito de lodo, bem como para avaliar o efeito das mudanças no posicionamento do sistema de distribuição do afluente na fluidodinâmica.

Essa divisão consistiu em uma zona primária (leito de lodo) próxima da região do sistema de distribuição do afluente, localizada na parte inferior do reator, e em uma zona secundária, que compreende o restante do reator, distante da região de entrada do afluente. Nas simulações foram considerados efeitos inerciais dos jatos de entrada na zona primária e efeitos convectivos na zona secundária.

\section{Avaliação dos sistemas de distribuição do afluente do reator}

As simulações foram realizadas em duas e três dimensões para os sistemas de distribuição do reator.

A configuração 1 possuía uma entrada para o afluente no centro da base do reator UASB utilizado por Carvalho et al. (2008), em sentido ascendente. Na configuração 2 foram consideradas duas entradas para o afluente à distância de $14 \mathrm{~cm}$ do centro da base do reator, em sentido ascendente. Na configuração 3, a posição do sistema de distribuição do afluente foi alterada para as laterais do reator. Na configuração 4 foram acrescentadas três entradas distantes $5 \mathrm{~cm}$ da base do reator, com fluxo em sentido descendente. Em todas as configurações avaliadas a tubulação do sistema de distribuição tinha 7,5 $\mathrm{mm}$ de diâmetro e volume útil de $105 \mathrm{~L}$ (cada).

Na Figura 2 são apresentadas as configurações de 1 a 4, com setas indicando o sentido do escoamento do fluxo do afluente, o leito de lodo e as malhas geradas automaticamente por meio da ferramenta computacional COMSOL Multiphysics para a realização das simulações. As malhas são compostas por aproximadamente 4.400 elementos triangulares para esse modelo bidimensional. A Figura 3 exibe a

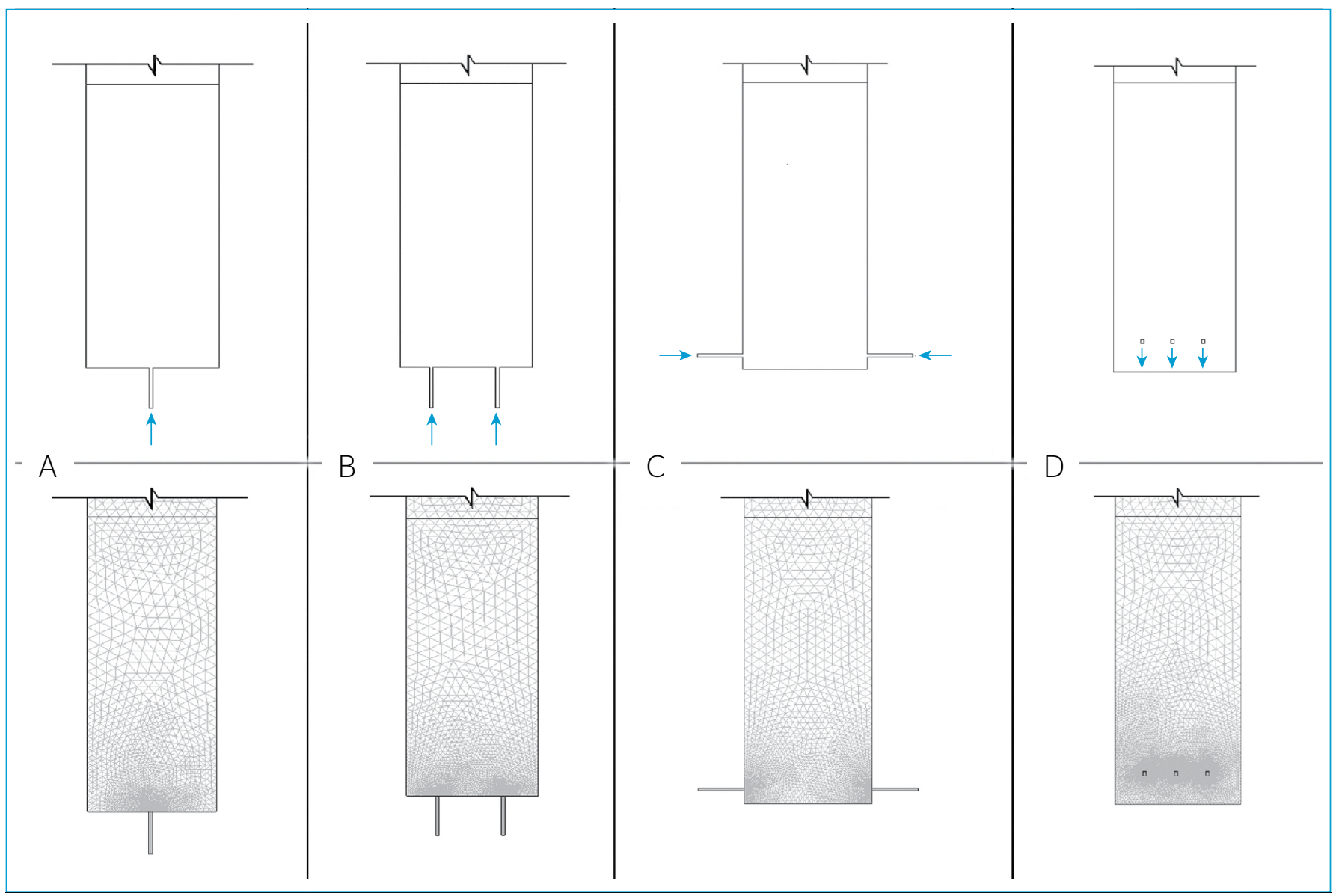

Figura 2 - Desenho esquemático do detalhe do sistema de distribuição do afluente e do leito de lodo das diferentes configurações de reatores bidimensionais propostas e a malha utilizada para as simulações: (A) configuração 1, (B) configuração 2, (C) configuração 3 e (D) configuração 4. 
mesma situação da Figura 2, exceto pela composição das malhas, que agora contemplam cerca de 70 mil elementos tetraédricos para esse modelo tridimensional.

As Figuras 2 e 3 expõem as malhas dos sistemas de distribuição do afluente e da região de contato entre as fases líquida e sólida, pois são regiões em que ocorrem mais variações das propriedades do escoamento.

As simulações bidimensionais e tridimensionais permitem visualizar as diferenças do comportamento dos escoamentos no plano, considerando o domínio espacial na região de entrada do reator.

$\mathrm{Na}$ Tabela 1 são delineados as condições de contorno e os parâmetros usados nas simulações dos modelos bidimensionais e tridimensionais, determinados com base nos estudos de Carvalho et al. (2008) e Ren et al. (2009).

\section{Modelo de fluidodinâmica computacional}

\section{Modelo Euler-Euler}

Neste trabalho foi utilizado um modelo de mistura de fluxo de duas fases para descrever o comportamento do escoamento em cada
Tabela 1 - Parâmetros utilizados nas simulações dos modelos de reatores anaeróbios de manta de lodo e fluxo ascendente bidimensionais e tridimensionais.

\begin{tabular}{l|c|c}
\multirow{2}{*}{ Parâmetros } & \multicolumn{2}{|c}{ Valores } \\
\cline { 2 - 3 } Condição de parede (wall) & nodelos 2D & Modelos 3D \\
\hline Condição de saída (outlet), Po & no slip \\
\hline Velocidade de entrada da fase contínua (inlet) & $0,1 \mathrm{~m} \cdot \mathrm{s}^{-1}$ & $0,1 \mathrm{~m} \cdot \mathrm{s}^{-1}$ \\
\hline Vazão da fase líquida na entrada & $16 \mathrm{~L} \cdot \mathrm{h}^{-1}$ & $16 \mathrm{~L} \cdot \mathrm{h}^{-1}$ \\
\hline Tempo de detenção hidráulica & $10 \mathrm{~h}$ & $10 \mathrm{~h}$ \\
\hline Densidade da fase contínua & $1.000 \mathrm{~kg} \cdot \mathrm{m}^{-3}$ & $1.000 \mathrm{~kg} \cdot \mathrm{m}^{-3}$ \\
\hline Viscosidade dinâmica da fase contínua & $0,001 \mathrm{~Pa} \cdot \mathrm{S}$ & $0,001 \mathrm{~Pa} \cdot \mathrm{s}$ \\
\hline Quantidade inicial de lodo & $35 \%$ & $35 \%$ \\
\hline Densidade da fase dispersa (lodo) & $1.460 \mathrm{~kg} \cdot \mathrm{m}^{-3}$ & $1.460 \mathrm{~kg} \cdot \mathrm{m}^{-3}$ \\
\hline Diâmetro das partículas de lodo & $0,001 \mathrm{~m}$ & $0,001 \mathrm{~m}$ \\
\hline Quantidade aproximada de elementos da malha & 4.400 & 70.000 \\
\hline
\end{tabular}

Po: pressão; 2D: duas dimensões; 3D: três dimensões

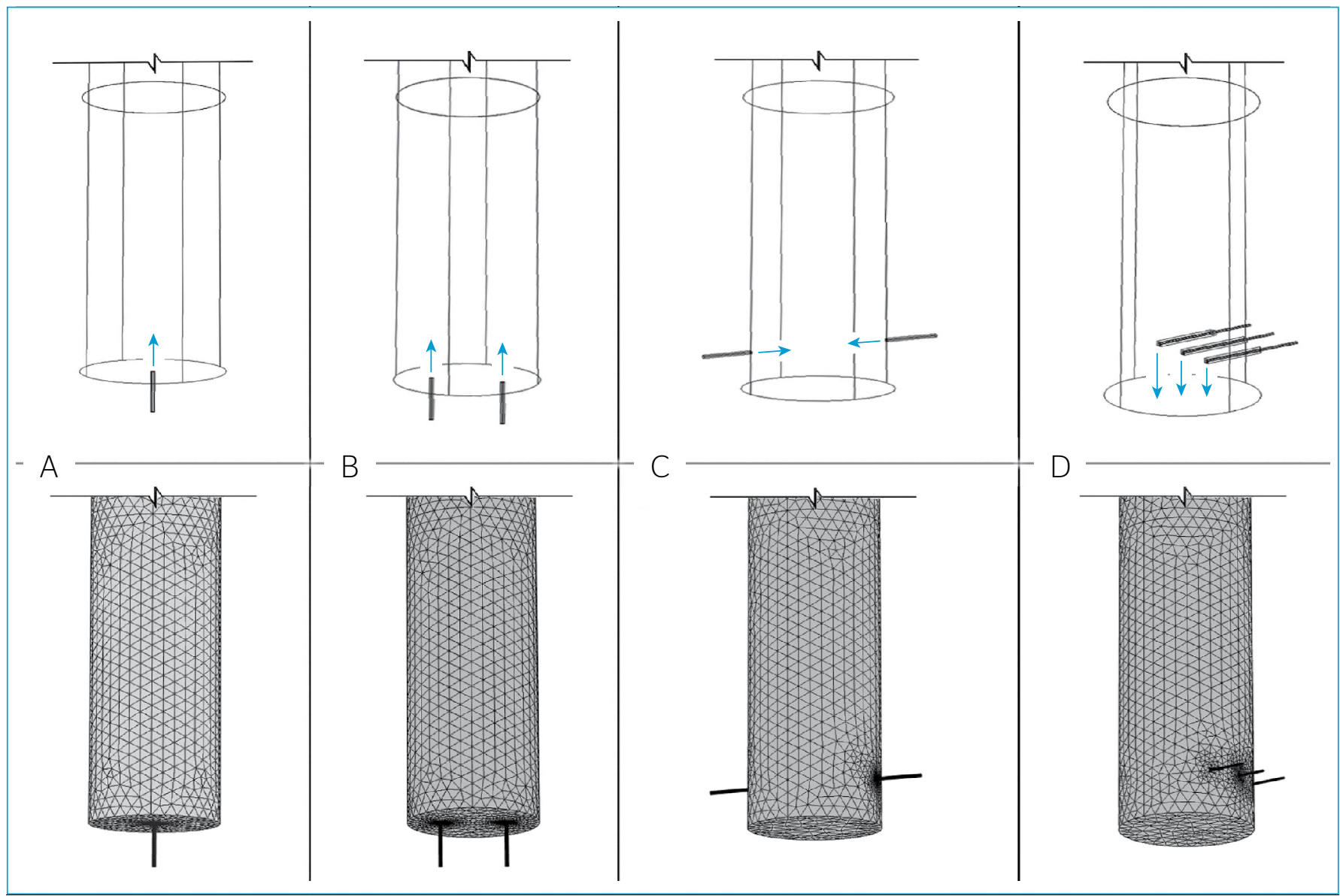

Figura 3 - Desenho esquemático do detalhe do sistema de distribuição do afluente e do leito de lodo das diferentes configurações de reatores tridimensionais propostas e a malha utilizada para as simulações: (A) configuração 1, (B) configuração 2, (C) configuração 3 e (D) configuração 4. 
uma, para realização das simulações no programa. Nesse modelo foi determinada a concentração média de cada fase, ou fração de volume, que é resolvida por um campo de velocidade para cada fase. O modelo contemplou uma fase dispersa com partículas sólidas (lodo anaeróbio) e uma fase contínua líquida (água residuária) simulada como água.

O modelo é fundamentado nos seguintes pressupostos: a densidade de cada fase é aproximadamente constante; ambas as fases possuem o mesmo campo de pressão; a velocidade relativa entre as duas fases é determinada essencialmente assumindo equilíbrio entre pressão, gravidade e arrasto viscoso.

$\mathrm{Na}$ condição inicial do escoamento, a água residuária foi considerada como água (fase dispersa, contínua) com densidade de 1.000 kg.m $\mathrm{m}^{-3}$ e viscosidade dinâmica de 0,001 Pa.s; e os grânulos do lodo foram de 0,001 m de diâmetro e densidade de $1.460 \mathrm{~kg} . \mathrm{m}^{-3}$, ocupando $35 \%$ da fração do volume até a altura de $0,70 \mathrm{~m}$ do reator, a partir da base do leito de lodo. Esses valores são os mesmos adotados por Ren et al. (2009).

\section{Equações governantes}

As principais equações que descrevem o processo são a equação dinâmica do momento (Equação 1) e a equação da continuidade (Equação 2), aplicadas à mistura das fases líquida e sólida.

$\rho \mathrm{u}_{\mathrm{t}+} \rho(\mathrm{u} \cdot \nabla) \mathrm{u}=-\nabla \mathrm{p}-\nabla \cdot\left(\rho \mathrm{c}_{\mathrm{d}}\left(1-\mathrm{c}_{\mathrm{d}}\right) \mathrm{u}_{\text {slip }} \cdot \mathrm{u}_{\text {slip }}\right)+\nabla \cdot \tau_{\mathrm{Gm}}+\rho \mathrm{g}+\mathrm{F}$

Em que:

$\rho=$ massa específica do fluido em $\mathrm{kg} \cdot \mathrm{m}^{-3}$;

$\mathrm{u}=$ velocidade em $\mathrm{m} \cdot \mathrm{s}^{-1}$;

$p=$ pressão em $\mathrm{Pa}$;

$c_{d}=$ fração de massa da fase dispersa em $\mathrm{kg} \cdot \mathrm{kg}^{-1}$;

$\mathrm{u}_{\text {slip }}=$ velocidade relativa entre as duas fases em $\mathrm{m} \cdot \mathrm{s}^{-1}$;

$\tau_{\mathrm{Gm}}=$ soma das tensões viscosas e turbulentas em kg.m $\mathrm{m}^{-1} \cdot \mathrm{s}^{-2}$;

$\mathrm{g}=$ vetor gravidade $\mathrm{em} \mathrm{m} \cdot \mathrm{s}^{-2}$;

$\mathrm{F}=$ forças de volume adicionais em N.m $\mathrm{m}^{-3}$.

$\rho_{\mathrm{t}}+\nabla \cdot(\rho \mathrm{u})=0$

Em que:

$\rho=$ massa específica do fluido em $\mathrm{kg} \cdot \mathrm{m}^{-3}$;

$\mathrm{u}=$ velocidade em $\mathrm{m} \cdot \mathrm{s}^{-1}$.

\section{Solução numérica}

As equações do modelo multifásico foram resolvidas utilizando a ferramenta computacional COMSOL Multiphysics pelo método de elementos finitos, considerando uma fase líquida (água residuária) e outra fase dispersa (lodo anaeróbio), contando com uma variedade de métodos numéricos que formam a base dos solvers disponíveis. Para a realização das simulações deste estudo, usou-se o solver Spooles, por apresentar mais rapidez de processamento.

De acordo com Simões et al. (2009), a solução do problema é calculada nos nós de cada elemento. Dessa maneira, governa-se a acurácia da solução do problema pelo número de elementos da malha gerada. Geralmente, quanto maior o número de elementos, maior será a acurácia, porém maior também será o custo computacional em tempo de processamento. As malhas grosseiras (com maior espaçamento entre os pontos) podem ser otimizadas pelo refinamento de seus elementos, originando uma malha com espaçamento pequeno entre os pontos.

As simulações numéricas foram realizadas em condições transientes, tendo em vista o período necessário para que o escoamento se tornasse completamente desenvolvido, como tempo de duração das simulações. Neste trabalho foi estabelecida duração total do escoamento de $600 \mathrm{~s}$.

O critério de convergência empregado pelo solver consistiu na utilização de tolerância relativa para os erros estimados. Considera-se que o algoritmo convergiu para solução correta quando o critério de convergência foi atingido, ou seja, quando o valor do erro estimado foi menor que $1.10^{-3}$, correspondente ao valor da tolerância relativa aplicado no presente trabalho.

Todas as simulações foram realizadas em um computador com um processador Intel i7, com 12 GB de memória RAM, e velocidade de $2,80 \mathrm{GHz}$.

\section{RESULTADOS E DISCUSSÃO}

Os resultados das simulações são apresentados na forma de figuras, nas quais são indicadas as linhas de corrente do fluxo e a velocidade de mistura da fase líquida com a sólida, nas diferentes configurações do sistema de distribuição do afluente dos reatores e no tempo final das simulações para as geometrias bidimensionais e tridimensionais. A velocidade da mistura é representada por vetores com tamanhos proporcionais a sua grandeza.

Na Figura 4 são exibidos as linhas de corrente da fase líquida e os vetores da velocidade de mistura ao final das simulações das configurações de entrada de reatores bidimensionais.

Por meio dos resultados da velocidade de mistura obtidos, verificou-se formação de caminhos preferenciais pelas aproximações das linhas de corrente e pelas dimensões dos vetores de velocidade, com maior grandeza ao longo do escoamento. A presença de caminhos preferenciais nas laterais do reator foi observada nas configurações 1 (Figura 4A) e 3 (Figura 4C) do sistema de distribuição do afluente.

De acordo com Teixeira et al. (2009), a presença de caminhos preferenciais na manta de lodo (lodo menos concentrado) e no leito de lodo (lodo mais concentrado) de reatores UASB acarreta diminuição 
do TDH e no tempo de contato entre a biomassa e o substrato, prejudicando sua eficiência.

$\mathrm{Na}$ Figura 4B foi constatada formação de vórtices na parte inferior do reator, demonstrando mistura eficiente do lodo na configuração 2 do sistema de distribuição do afluente. Esse comportamento também foi encontrado na configuração 4 (Figura 4D). Nessa configuração foi observada melhor sedimentação do lodo pelo número de recirculações, sentido e intensidade dos vetores de velocidade de mistura na região.

A distribuição adequada da água residuária é importante, pois uma boa condição de mistura, ocasionada pelo fluxo ascensional de líquido e das bolhas de gás, proporciona o contato ótimo do substrato com o lodo presente no reator, evitando caminhos preferenciais (VERSIANI, 2005).

As malhas utilizadas para as simulações tridimensionais foram refinadas, pois uma boa solução numérica depende de uma malha de pontos de boa qualidade e que seja refinada em regiões com grandes variações das propriedades do escoamento, de acordo com Fortuna (2000). A simulação tridimensional permite melhor representar espacialmente os escoamentos, tendo em vista que os fenômenos de recirculação, zonas mortas e caminhos preferenciais ocorrem nas três direções no interior do reator.

A simulação tridimensional diferencia-se da bidimensional, por apresentar as linhas de corrente de forma espacial, e ainda permite realizar a quantificação volumétrica das regiões de zonas mortas.
Na Figura 5 estão as linhas de corrente da fase líquida e os vetores da velocidade de mistura ao final das simulações das configurações de entrada de reatores tridimensionais. A formação de caminhos preferenciais no interior dos reatores é verificada nos resultados da velocidade de mistura obtidos para as configurações 1, 2 e 3 do sistema de distribuição do afluente, conforme Figura 5.

Nas simulações do reator foram notados a formação de caminhos preferenciais na parte central na configuração 1 (Figura 5A), dois caminhos centrais na configuração 2 (Figura 5B) e dois caminhos nas laterais na configuração 3 (Figura 5C).

Na configuração 4 (Figura 5D) não foi vista formação de caminhos preferenciais, possibilitando melhor distribuição do afluente no interior do reator. Também foi achado melhor perfil de mistura do lodo com a fase líquida, demonstrado pela formação de vórtices na parte inferior do reator, na qual o lodo anaeróbio está mais concentrado.

López-Jiménez et al. (2015) investigaram as características do fluxo de lodo em um digestor anaeróbio empregado no tratamento de águas residuárias da cidade de Valência, Espanha. As simulações foram realizadas para o digestor de fluxo descendente com duas entradas na parte superior, com diferentes angulações, por um estudo numérico em três dimensões. Os autores relatam que, quanto mais circunferencial foi o ângulo de distribuição do fluido na entrada, menor era a incidência de curtos-circuitos hidráulicos e de zonas mortas, com poucas mudanças na geometria do reator. Segundo os autores, essas

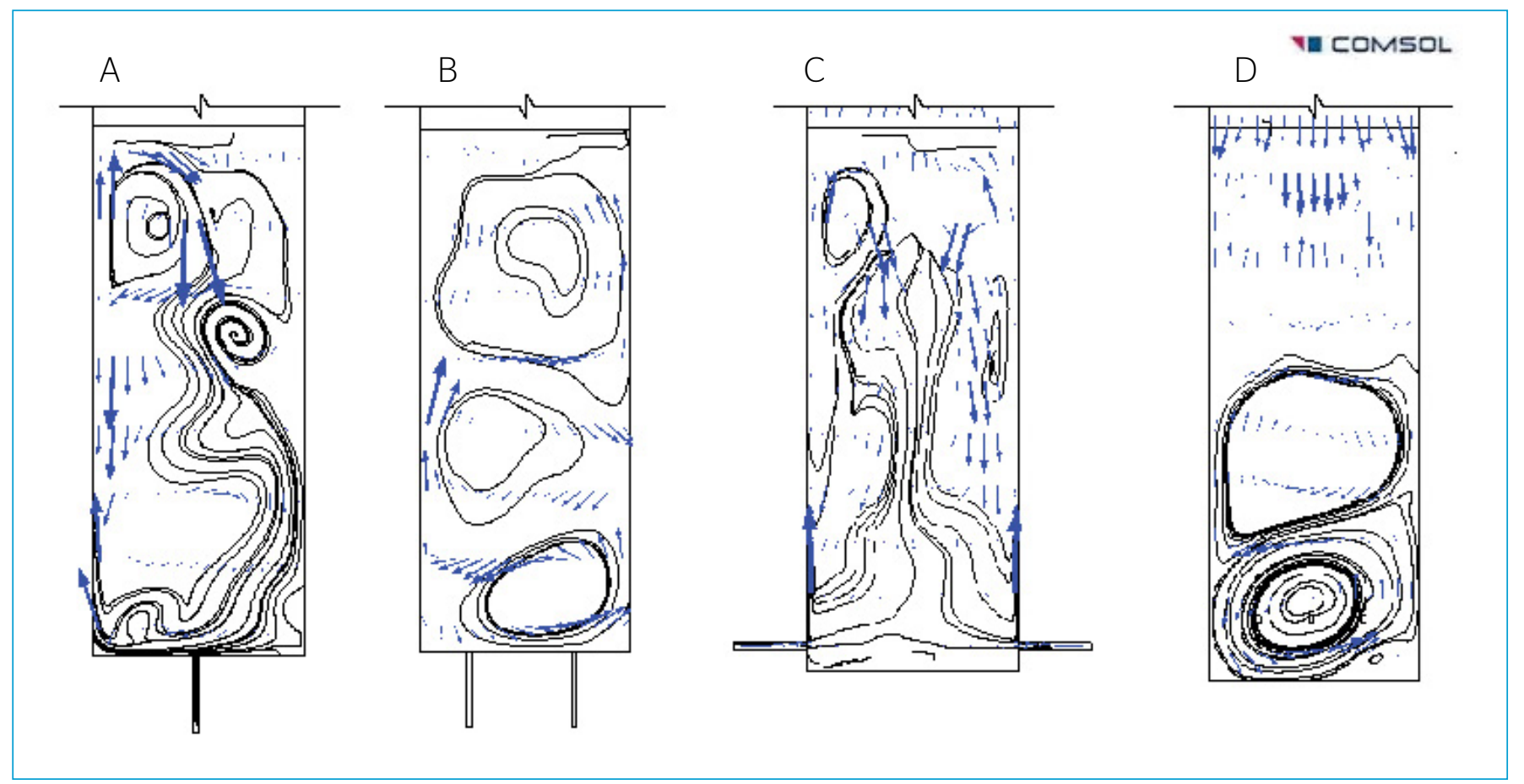

Figura 4 - Linhas de corrente da fase líquida e vetores da velocidade de mistura ao final das simulações das configurações de entrada de reatores bidimensionais: (A) configuração 1, (B) configuração 2, (C) configuração e (D) configuração 4. 
conclusões demonstraram que, quanto mais tangencial é o ângulo na entrada, menores são os volumes de zonas mortas e curtos-circuitos hidráulicos ou recirculações no reator.

De acordo com Sartori et al. (2015), as zonas estagnadas são regiões do reator em que os tempos de residência das partículas do fluido são relativamente altos, quando comparados ao DTR teórico.

Ren et al. (2009) consideram as zonas estagnadas como regiões nas quais a velocidade da fase líquida é inferior a $5 \%$ da velocidade ascensional média. Com base nesse pressuposto, foram calculados os volumes de zonas estagnadas para os modelos tridimensionais, conforme Figura 6.

$\mathrm{Na}$ Figura 6 é possível notar que a partir de $40 \mathrm{~s}$ de simulação o escoamento se torna completamente desenvolvido, e após esse tempo foram observados os maiores volumes de zonas estagnadas encontradas em cada configuração.

As porcentagens máximas dos volumes de zonas estagnadas em relação ao volume total do reator foram: $4 \%$ na configuração 1, $2 \%$ nas configurações 2 e 3, e 3\% na configuração 4 . Esses valores foram inferiores aos obtidos por Singh et al. (2006), de 10\% em um reator UASB em escala de bancada $(8 \mathrm{~L})$ operado a $32^{\circ} \mathrm{C}$ e de $11 \%$ no mesmo reator operado a $20^{\circ} \mathrm{C}$, e inferiores aos verificados por Wu et al. (1992), de 25 e 35\% em um reator UASB de 3,1 L. Esses autores realizaram ensaios de estímulo-resposta com injeção do traçador em entrada única do afluente na lateral do reator.

Bitog et al. (2014) observaram que a porcentagem do volume de zonas mortas é influenciada pela posição e geometria dos defletores em fotobiorreatores do tipo coluna de bolhas cilíndricas (PBRS), com entrada única, por meio da CFD em 32 simulações numéricas. Os autores concluíram que a taxa de fluxo de ar, o diâmetro e a geometria dos

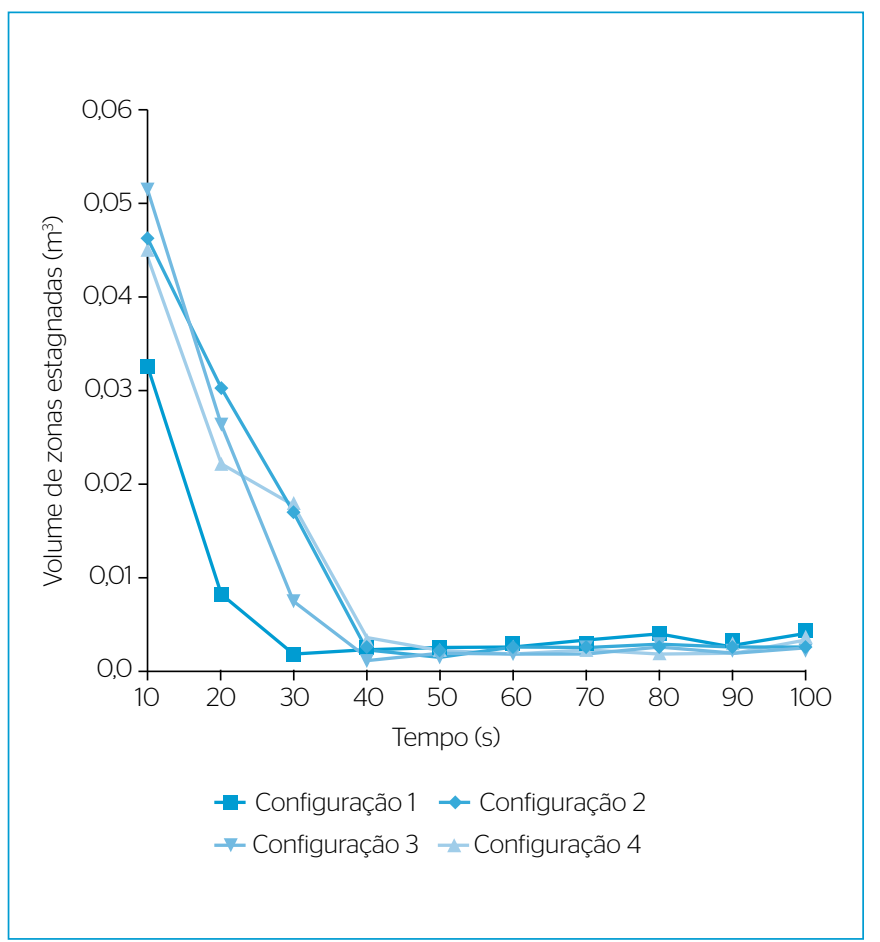

Figura 6 - Volume de zonas estagnadas em relação ao tempo de simulação para diferentes configurações do sistema de distribuição do afluente dos reatores.

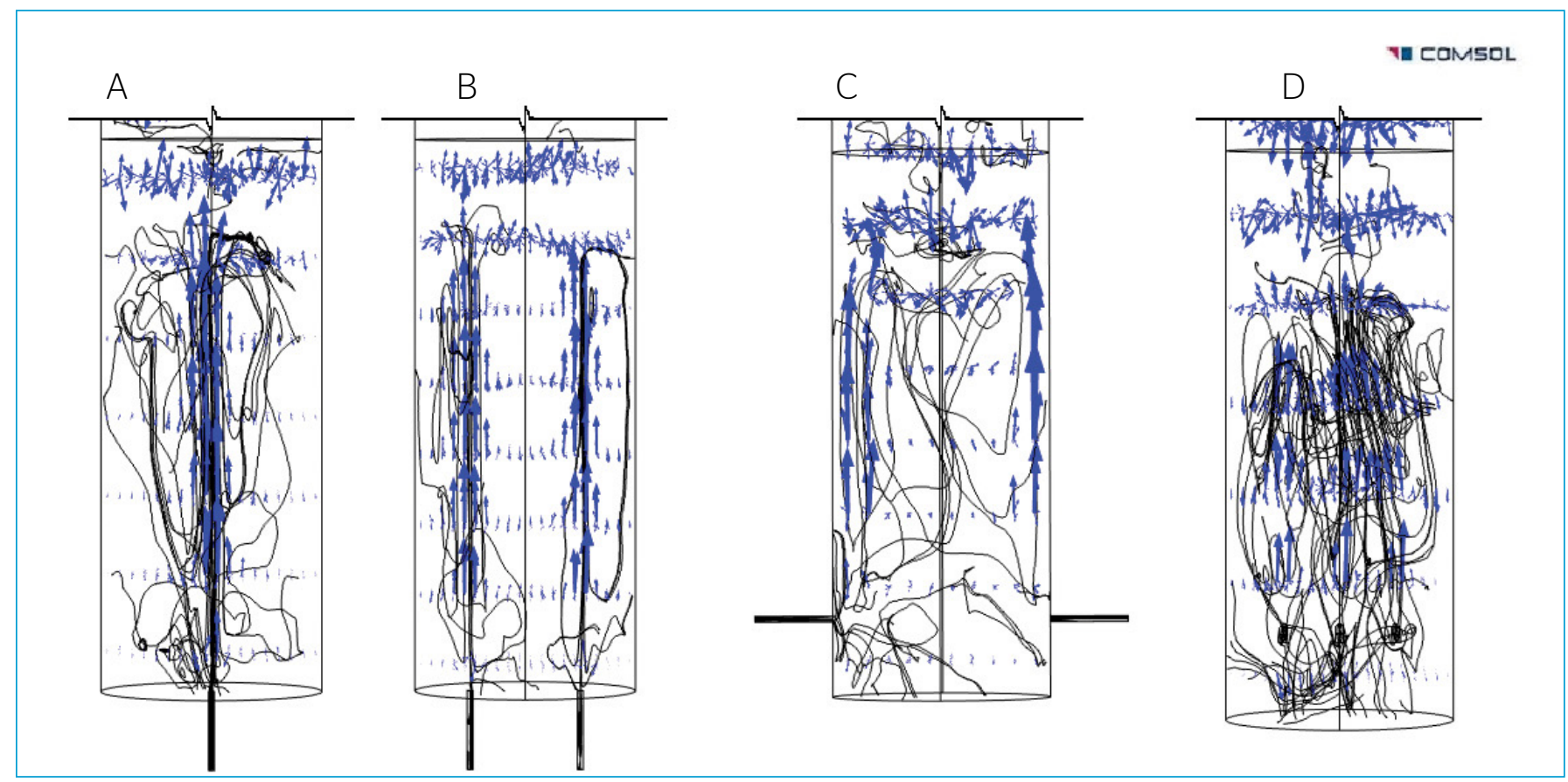

Figura 5 - Linhas de corrente da fase líquida e vetores da velocidade de mistura ao final das simulações das configurações de entrada de reatores tridimensionais: (A) configuração 1, (B) configuração 2, (C) configuração 3 e (D) configuração 4. 
defletores interferem significativamente no volume de zonas mortas e que a maior parte das zonas mortas foi localizada na parte inferior e perto das paredes internas do fotobiorreator.

\section{CONCLUSÕES}

Nas simulações bidimensionais e tridimensionais realizadas neste trabalho, constataram-se:

- melhor distribuição do afluente na configuração 4 do reator UASB, que contempla três entradas para o afluente em fluxo descendente. Nessa configuração foi notada mistura do lodo com a fase líquida, demonstrada pela formação de vórtices na parte inferior do reator, com maior concentração do lodo anaeróbio;
- identificação de intensa formação de caminhos preferenciais nas laterais do reator na configuração 3, que contém duas entradas laterais, apontando a ineficiente mistura do afluente com o lodo anaeróbio;

- porcentagem de zonas estagnadas no reator tipo UASB de $3 \%$ na configuração 4 , superior àquela observada de $2 \%$ nas configurações 2 e 3 , apesar de as simulações com a configuração 4 indicarem as melhores características hidrodinâmicas.

\section{AGRADECIMENTOS}

Os autores agradecem ao órgão fomentador: Conselho Nacional de Desenvolvimento Científico e Tecnológico (CNPq), por meio do Edital Jovem Pesquisador 571535/2008-9 e Edital Universal 484426/2011-7.

\section{REFERÊNCIAS}

BITOG, J. P. P.; LEE, I. B.; OH, H. M.; HONG, S. W.; SEO, I. H.; KWON, K. S. (2014). Optimised hydrodynamic parameters for the design of photobioreactors using computational fluid dynamics and experimental validation. Biosystems engineering, v. 122, p. 42-61.

CARVALHO, K. Q.; SALGADO, M. T.; PASSIG, F. H.; PIRES, E. C. (2008). Avaliação hidrodinâmica de reator UASB submetido a variação cíclica de vazão. Revista Engenharia Sanitária e Ambiental, v. 13, n. 2. p. 226-235.

COMSOL Multiphysics:. (2010). Manual do usuário do software. Módulo de Engenharia Química, versão 4.0.

FOGLER, H. S. (1999). Elements of chemical reaction engineering. 3. ed. Rio de Janeiro: Prentice-Hall Education. 976 p.

FORTUNA, A. O. (2000). Técnicas computacionais para dinâmica de fluidos: conceitos básicos e aplicações. São Paulo: Edusp. 426 p.

ISLABÃO, G. I.; PINTO, J. C. C. S.; VIANNA JÚNIOR, A. S. (2O10). Technological trends in CFD applications. Journal of Technology Management \& Innovation, v. 5, n. 2, p. 76-83.

LAURENT, J.; SAMSTAG, R. W.; DUCOSTE, J. M.; GRIBORIO, A.; NOPENS, I.; BATSTONE, D. J.; WICKS, J. D.; SAUNDERS, S.; POTIER, O. (2014). A protocol for the use of computational fluid dynamics as a supportive tool for wastewater treatment plant modelling. Water Science \& Technology, v. 70, n. 10, p. 1575-1584. DOI: 10.2166/ wst.2014.425

LEVENSPIEL, O. (2000). Engenharia das reações químicas. 3. ed. São Paulo: Edgard Blucher. 563 p.

LÓPEZ-JIMÉNEZ, P. A.; ESCUDERO-GONZÁLEZ, J.; MARTÍNEZ, T. M.; MONTAÑANA, V. F.; GUALTIERI, C. (2015). Application of CFD methods to an anaerobic digester: The case of Ontinyent WWTP, Valencia, Spain. Journal of Water Process Engineering, v. 7. p. 131-140.
MAESTRI, R. D. \& GIMENEZ, J. R. (1998). Hydrodynamic study of up flow anaerobic sludge blanket (UASB) reactors. In: Latin-American Workshop-Seminar Wastewater Anaerobic Treatment. Anais... Viña del Mar, Chile, v. 1, p. 1-11.

MIRANDA, C. B. N.; DUQUE, G. W. D.; SILVA, J. A.; TEIXEIRA, F. N. (2010) Simulação numérica do escoamento em um ciclone separador usando CFD. In: SIMPÓSIO DE MECÂNICA COMPUTACIONAL, 9., São João del-Rei. Anais... São João del-Rei: ABMEC, v. 1, p. 1-4.

REN, T. T.; MU, Y.; NI, B. Y.; YU, H. Q. (2009). Hydrodynamics of upflow anaerobic sludge blanket reactors. Alche Journal: Environmental and Energy Engineering, v. 55, n. 2, p. 516-528.

SARTORI, M.; OLIVEIRA, D. S.; TEIXEIRA, E. C.; RAUEN, W. B.; REIS, N. C. (2015). CFD modelling of helically coiled tube flocculators for velocity gradient assessment. Journal of the Brazilian Society of Mechanical Sciences and Engineering, v. 37, p. 187-198.

SIMÕES, M. R.; MONTOJOS, B. G.; MOURA, N. R.; SU, J. (2009) Validation of turbulence models for simulation of axial flow compressor. In: INTERNATIONAL CONGRESS OF MECHANICAL ENGINEERING, 2O., Gramado. Proceedings... Rio de Janeiro: ABCM.

SINGH, K. S.; VIRARAGHAVAN, T; BHATTACHARYYA, D. (2006). Sludge blanket height and flow pattern in UASB reactors: temperature effects. ASCE Journal of Environmental Engineering, v. 132, n. 8, p. 895-900.

TEIXEIRA, A. R.; CHERNICHARO, C. A. L.; SOUTO, T. F. S.; PAULA, F. S. (2009). Influência da alteração da distribuição do tamanho de partículas no desempenho de reatores UASB tratando esgotos domésticos. Revista Engenharia Sanitária e Ambiental, v. 14, n. 2, p. 159-166.

VERSIANI, B. M. (2005). Desempenho de um reator UASB submetido a diferentes condições operacionais tratando esgotos sanitários do Campus da Universidade Federal do Rio de Janeiro. Dissertação (Mestrado em Ciências em Engenharia Civil) Universidade Federal do Rio de Janeiro, Rio de Janeiro. 
VERSTEEG, H. K. \& MALALASEKERA, W. (1995). An introduction to computational fluid dynamics: the finite volume method. Harlow: Ed. Longman Scientific and Technical. 255 p.

WANG, X.; DING, J.; GUO, W. Q.; REN, N. Q. (2010). A hydrodynamicsreaction kinetics coupled model for evaluating bioreactors derived from CFD simulation. Bioresource Technology, v.101, p. 9749-9757.
WANG, X.; DING, J.; REN, N. Q.; LIU, B. F.; GUO, W. Q. (2009). CFD simulation of an expanded granular sludge bed (EGSB) reactor for biohydrogen production. International Journal of Hidrogen Energy, China, p. 9686-9695.

WU, M. M.; VOICE, T. C.; HICKEY, R. F. (1992). Hydraulic characteristics of an upflow anaerobic sludge blanket UASB reactor with granular sludge. In: INDUSTRIAL WASTE CONFERENCE, 47., West Lafayette. Proceedings... West Lafayette: Lewis, Boca Raton. 